\title{
Risk of hip fracture in patients with a history of schizophrenia
}

\author{
LOUISE HOWARD, GRAHAM KIRKWOOD and MORVEN LEESE
}

\section{Background There is evidence of an association between decreased bone mineral density, schizophrenia, and prolactin-raising antipsychotic medication. However, it is not known whether this is clinically significant.}

Aims To investigate whether patients with a history of schizophrenia are at increased risk of hip fracture.

Method In a case-control study, we compared cases of 'hip fracture' on the General Practice Research Database $(n=16341)$ with matched controls $(n=29889)$.

Results Hip fracture was associated with schizophrenia $(\mathrm{OR}=1.73 ; 95 \% \mathrm{Cl}$ I.32-2.28), and prolactin-raising antipsychotics (OR=2.6; 95\% Cl 2.432.78), in the univariate analysis. In the multivariate analysis, prolactin-raising antipsychotics were independently associated with hip fracture but schizophrenia was not. A significant interaction between gender and antipsychotics was found in the association with hip fracture $(P=0.042) ; O R=2.12$ (95\% Cl I.73-2.59) for men, $O R=1.93$ (95\% Cl .78-2.10) for women.

Conclusions The association between prolactin-raising antipsychotic medication and hip fracture may have serious implications for public health. Mental health service patients may require preventive measures including dietary and lifestyle advice.

Declaration of interest None. Funding detailed in Acknowledgements.
A growing number of recent reports have suggested that there is an association between decreased bone mineral density and schizophrenia (Halbreich et al, 1995; Abraham et al, 2003), particularly in patients treated with psychotropic medication (Halbreich \& Palter, 1996; O’Keane $\&$ Meaney, 2005). However, these studies have not examined whether this is clinically significant, leading to an increased risk of fractures. The prevalence of functional psychoses is 4 per 1000 (Jenkins et al, 1997), with approximately half of that figure relating to schizophrenia. The identification of schizophrenia as a risk factor for osteoporosis would therefore have important public health implications.

Hip fracture is the most important fracture in terms of patient morbidity and mortality and for utilisation of health service resources (Cumming et al, 1997). As fracture is common in the older population, a small increase in the risk of fracture associated with psychotic disorders could have a considerable public health effect. We therefore chose to investigate whether there was an association between schizophrenia and hip fractures, using a case-control study design with data from a UK primary care data-set, the General Practice Research Database (GPRD). Our hypothesis was that patients with a history of schizophrenia would have a significantly increased risk of antipsychotic-induced osteoporotic hip fractures compared with a control group matched for age and general practice.

\section{METHOD}

\section{Data source}

The GPRD was set up in the UK in 1987 and contains the computerised medical records of approximately $5 \%$ of the UK population in primary care (Walley \& Mantgani, 1997). Data recorded include prescription details, clinical events, preventive care provided, specialist referrals, hospital admissions and their major outcomes. The data collected are audited regularly and the participating general practices subjected to a number of quality checks, including internal validation by crosschecking within practices and by comparisons with national statistics (Walley \& Mantgani, 1997). Only practices that comply with this quality control (i.e. are 'up-to-research standard') are retained within the database. The data are representative of the general population (Walley $\&$ Mantgani, 1997), although there is a bias towards larger group practices. Diagnoses of schizophrenia recorded on the GPRD have been validated in several studies $(\mathrm{Na}-$ zareth et al, 1993; Howard et al, 2002) and hip fracture recording is reported to be particularly complete (van Staa et al, 2001).

\section{Case-control analysis}

Stata version 8.2 for Windows was used for statistical analysis. All patients registered on the GPRD between 1 August 1987 and 22 November 1999 with a recorded diagnosis of fractured neck of femur or 'hip fracture' were identified, and designated as cases. Two controls per case were identified, matching on age, gender, general practice and duration of available GPRD data. For statistical efficiency a 1:1 ratio of cases to controls is ideal when the number of cases can be chosen. In our study, since the number of cases was limited, the number of controls was increased to two per case in order to achieve adequate power. Each case was assigned a date of diagnosis, defined as the date of the first hip fracture, and matching control individuals were assigned an identical 'pseudo' date of diagnosis. Only records that were up to research standard were used.

Variables included the patients' medical and psychiatric history, medication history and demographic details, as well as lifestyle factors (alcohol consumption, smoking and body mass index). All recorded diagnoses of schizophrenia were extracted and recorded for each case and control on an ever/never basis. Ever having had a prescription for a neuroleptic drug prior to or on the day of the first fracture was extracted and recorded. Where comorbid disorders were examined all disorders under the main relevant ICD-9 heading were included e.g. 'intestinal diseases' includes all diseases under this heading in ICD-9 (World Health Organization, 1978). To 
Table I Associations between clinical and drug variables and hip fracture, General Practice Research Database, $1987-1999$

\begin{tabular}{|c|c|c|c|c|c|c|c|c|}
\hline \multirow[t]{2}{*}{ Exposure } & \multicolumn{2}{|c|}{$\begin{array}{c}\text { Cases } \\
(n=16341)\end{array}$} & \multicolumn{2}{|c|}{$\begin{array}{c}\text { Controls } \\
(n=29889)\end{array}$} & \multicolumn{2}{|c|}{$\begin{array}{c}\text { Univariate } \\
\text { analysis }\end{array}$} & \multicolumn{2}{|c|}{$\begin{array}{l}\text { Bivariate } \\
\text { analysis }\end{array}$} \\
\hline & $n$ & (\%) & $n$ & (\%) & OR & $(95 \% \mathrm{Cl})$ & $\begin{array}{c}\text { OR for each } \\
\text { variable adjusting } \\
\text { for schizophrenia }\end{array}$ & $\begin{array}{c}\text { OR for } \\
\text { schizophrenia } \\
\text { adjusting for } \\
\text { each variable }\end{array}$ \\
\hline Schizophrenia diagnosis & 100 & $(0.61)$ & 110 & $(0.37)$ & 1.73 & $(1.32-2.28)$ & & \\
\hline Any neuroleptic & 2246 & $(13.74)$ & 1779 & $(5.95)$ & 2.60 & $(2.43-2.78)$ & 2.60 & 1.00 \\
\hline Any SSRI ${ }^{2}$ & 955 & $(5.84)$ & 892 & $(2.98)$ & 2.01 & $(I .82-2.2 I)$ & 2.01 & 1.70 \\
\hline Any anticonvulsant ${ }^{3}$ & 869 & $(5.32)$ & 805 & $(2.69)$ & 2.01 & $(1.82-2.22)$ & 2.01 & 1.70 \\
\hline Any tricyclic & 2905 & $(17.78)$ & 3259 & $(10.90)$ & 1.78 & $(1.69-1.89)$ & 1.78 & 1.69 \\
\hline Any hypnotic & 4195 & $(25.67)$ & 5149 & $(17.23)$ & 1.68 & $(1.60-1.77)$ & 1.68 & 1.62 \\
\hline \multicolumn{9}{|l|}{ Smoking status } \\
\hline Current smoker & 1304 & (7.98) & 1587 & $(5.31)$ & $1.49^{5}$ & $(1.37-1.62)$ & 1.49 & 1.75 \\
\hline Ex-smoker & 469 & $(2.87)$ & 834 & $(2.79)$ & $1.03^{5}$ & $(0.91-1.16)$ & 1.03 & 1.75 \\
\hline Hormone replacement therapy (women only) & 220 & $(I .7 I)$ & 488 & $(2.08)$ & 0.79 & $(0.66-0.94)$ & 0.79 & 1.60 \\
\hline \multicolumn{9}{|l|}{ Body mass index ${ }^{6}$} \\
\hline Underweight & 395 & $(2.42)$ & 232 & $(0.78)$ & $2.41^{6}$ & $(2.02-2.86)$ & 2.40 & 1.78 \\
\hline Overweight & 1144 & $(7.00)$ & 2839 & $(9.50)$ & $0.55^{6}$ & $(0.51-0.60)$ & 0.55 & 1.78 \\
\hline Obese & 322 & $(1.97)$ & 1136 & $(3.80)$ & 0.396 & $(0.34-0.44)$ & 0.39 & 1.78 \\
\hline
\end{tabular}

I. Any of chlorpromazine, thioridazine, droperidol, flupentixol, fluphenazine, haloperidol, pimozide, promazine, sulpiride, trifluoperazine, zuclopenthixol acetate and dihydrochloride as well as depot versions of flupentixol, fluphenazine, haloperidol, zuclopenthixol and pipotiazine.

2. Selective serotonin reuptake inhibitor: any of citalopram, fluoxetine, fluvoxamine, paroxetine and sertraline.

3. Any of carbamazepine, phenytoin, sodium valproate, lamotrigine and gabapentin.

4. Alcohol level compared with intake within recommended limits, defined as no more than 21 units a week for men and 14 units a week for women.

5. Smoking status compared with non-smoker.

6. Compared with normal body mass index defined as $18.5-24.9 \mathrm{~kg} / \mathrm{m}^{2}$.

enable all the data to be used, a category 'missing' was created for each variable where needed. Imputation would have added no extra information, since all the variables available to make imputations were already included in the analysis.

Variables that had been previously identified in the research literature as being significantly associated with hip fracture were examined in univariate analyses and those that were significantly associated in this analysis were selected for multivariate analysis. Conditional logistic regression was used to calculate an unadjusted odds ratio for each selected variable. The selected variables were added one by one into bivariate models to identify potential confounders with the schizophrenia diagnosis variable and to see how much, if at all, they reduced or increased the odds ratio obtained with the schizophrenia diagnosis variable alone. A multivariable model was fitted using conditional logistic regression in a forward stepwise process using likelihood ratio tests. Variables that had become non-significant were removed and again the fit of the model was tested. By including interaction terms, gender and age at the time of the first fracture were tested to assess for modification of the effect of the schizophrenia diagnosis and of ever having had a prescription for neuroleptic medication on the incidence of hip fracture.

Ethical approval was granted by the Scientific Advisory and Ethical Group at the Medicines Control Agency, who are responsible for ethical issues for all projects using the GPRD.

\section{RESULTS}

There were 16341 cases of hip fracture and 29889 controls. There were two controls per case for 13548 of the cases and one control per case for the remaining 2793 . The mean age of the cases and controls at the time of first fracture was 79 years (s.d.=12), and $12854(79 \%)$ of the cases were female. Variables identified as having an association with hip fracture in the univariate analysis included cerebrovascular disease $(\mathrm{OR}=1.89,95 \%$ CI $1.76-2.03)$, blood disorders $(\mathrm{OR}=1.85$, 95\% CI $1.73-$ 1.97), intestinal disorders $(\mathrm{OR}=1.7,95 \%$ CI 1.69-1.86), eye disorders $(\mathrm{OR}=1.11$, $95 \%$ CI 1.06-1.17), ear disorders $(\mathrm{OR}=1.02$, 95\% CI $0.97-1.08)$, urinary disorders $(\mathrm{OR}=1.50,95 \%$ CI $1.42-1.57)$, laxatives $(\mathrm{OR}=1.97,95 \% \mathrm{CI} 1.89-2.07)$, opioid analgesics $(\mathrm{OR}=1.94,95 \%$ CI 1.81-2.07), non-opioid analgesics $(\mathrm{OR}=1.85$, 95\% CI 1.77-1.93), inhaled corticosteroids $(\mathrm{OR}=1.26,95 \%$ CI $1.17-$ 1.36) and injected corticocosteroids $(\mathrm{OR}=1.07,95 \%$ CI 0.96-1.19). More details of variables of particular interest to this study, including psychotropic medications, are presented in Table 1. Medication with heparin was considered for inclusion in the analysis, but this was ruled out as only 8 cases and 5 controls were exposed to this drug. Fitting bivariate models for each of these variables along with the schizophrenia variable identified only one having a substantial effect on the odds ratio attached to schizophrenia diagnosis, namely having ever had a prescription for 
Table 2 Adjusted odds ratios for hip fractures in women: General Practice Research Database 1987-1999 $(n=36330)$

\begin{tabular}{|c|c|c|c|}
\hline Exposure & OR & $(95 \% \mathrm{Cl})$ & $P$ \\
\hline Schizophrenia diagnosis & 1.01 & $0.72-1.40$ & 0.971 \\
\hline Any neuroleptic ' & 1.93 & $1.78-2.10$ & $<0.001$ \\
\hline \multicolumn{4}{|l|}{ Body mass index ${ }^{2}$} \\
\hline Underweight & 2.17 & $1.78-2.64$ & $<0.001$ \\
\hline Overweight & 0.55 & $0.50-0.62$ & $<0.001$ \\
\hline Obese & 0.35 & $0.30-0.41$ & $<0.001$ \\
\hline Any SSRI & 1.24 & $1.10-1.40$ & $<0.001$ \\
\hline Any laxative & 1.36 & I.29-I.44 & $<0.001$ \\
\hline Any anticonvulsant & 1.39 & $1.23-1.57$ & $<0.001$ \\
\hline Opioid analgesics & 1.38 & I.27-I.50 & $<0.001$ \\
\hline Cerebrovascular disease & 1.48 & $1.36-1.62$ & $<0.001$ \\
\hline Blood diseases & 1.51 & $1.40-1.63$ & $<0.001$ \\
\hline Non-opioid analgesics & 1.47 & $1.39-1.55$ & $<0.001$ \\
\hline Any tricyclic & 1.16 & $1.09-1.25$ & $<0.001$ \\
\hline Any hypnotic & 1.16 & $1.10-1.23$ & $<0.001$ \\
\hline Diseases of urinary system & 1.17 & I.10-I.24 & $<0.001$ \\
\hline \multicolumn{4}{|l|}{ Smoking status ${ }^{3}$} \\
\hline Current smoker & 1.28 & $\mathrm{I} .14-1.43$ & $<0.001$ \\
\hline Ex-smoker & 1.00 & $0.85-1.19$ & 0.971 \\
\hline Hormone replacement therapy & 0.62 & $0.5 \mathrm{I}-0.75$ & $<0.001$ \\
\hline Inhaled corticosteroids & I.II & $\mathrm{I} .0 \mathrm{I}-\mathrm{I} .23$ & 0.035 \\
\hline Hypertensive disease & 0.87 & $0.82-0.93$ & $<0.001$ \\
\hline Ear diseases & 0.91 & $0.85-0.96$ & 0.002 \\
\hline
\end{tabular}

SSRI, selective serotonin reuptake inhibitor.

I. Neuroleptic medication prescribed before or on day of fracture.

2. Compared with normal body mass index defined as $18.5-24.9 \mathrm{~kg} / \mathrm{m}^{2}$.

3. Smoking status compared with non-smoker.

a neuroleptic. There was a significant association between having had more than one fall and hip fracture $(\mathrm{OR}=3.79,95 \% \mathrm{CI}$ 3.43-4.19). However, this was considered to be potentially on the causal pathway between schizophrenia diagnosis (and subsequent neuroleptic medication prescription) and hip fracture, assuming such an association exists, and consequently was not included in further analysis.

Although schizophrenia was significantly associated with hip fractures in the univariate analysis, this association was not significant when antipsychotic medication was added to a multivariate model as a potential confounder. A significant interaction was found between gender and having ever had a prescription for neuroleptic medication $\left(\chi^{2}=4.15\right.$, d.f. $=1, P=0.042$, likelihood ratio test). No such interaction was found for schizophrenia diagnosis. The model was therefore fitted separately for women (Table 2) and men (Table 3). There were some differences between the two gender-based models. Only in the female model were diseases of the urinary system, having ever had inhaled corticosteroids and diseases of the ear and mastoid process found to be significant. Only in the male model were alcohol consumption above recommended levels and diseases of the intestines and peritoneum found to be significant.

Dementia had been diagnosed in 3461 patients of this data-set $-1815(\mathbf{1 1 \%})$ cases and $1646(6 \%)$ controls; $\chi^{2}=478.36$, d.f. $=1, P<0.01$. Antipsychotic medication could have been prescribed for behavioural disturbance in dementia, so patients receiving such medication were excluded and the analyses repeated: this made negligible difference to any result (further details available from the authors upon request). The analyses were repeated using the 'cluster' option in Stata, but there was no evidence of clustering of fractures by general practice (further information available from the authors upon request).
There was also a significant interaction between age at the time of the first fracture (categorised) and having ever had a prescription for neuroleptic medication $\left(\chi^{2}=28.27\right.$, d.f. $=3, P<0.001$, likelihood ratio test). The interaction was most marked for those aged under 85 years $(n=31071$; OR for neuroleptic medication 2.29, 95\% CI 2.06-2.53) compared with those aged 85 years and over $(n=15113$; OR for neuroleptic medication $1.66,95 \%$ CI 1.49-1.85). Since there was an interaction for both age and gender, the model was also fitted separately with the data-set stratified by both these variables into women under 85 years old $(n=23289$; OR for neuroleptic medication $2.31,95 \%$ CI 2.06-2.58); women 85 years or older $(n=13027 ; \quad \mathrm{OR}=1.65,95 \%$ CI 1.47 $1.86)$; men under 85 years old $(n=7788$; $\mathrm{OR}=2.42$, 95\% CI 1.89-3.09); and men 85 years old or older $(n=2098$; OR $=2.12$, 95\% CI 1.51-2.99). Odds ratios were calculated for each of the individual neuroleptic medications which went to make up the combined neuroleptic variable, and are presented in Table 4. Also included here are figures for prochlorperazine, which was not included as part of the combined neuroleptic variable as it was felt that this might have been used largely as an anti-emetic. Atypical antipsychotic medications (which were only prescribed in the last few years of this data-set), were also considered separately, as there were too few prescribed to include in a combined antipsychotic variable, and odds ratios for these are also presented.

\section{DISCUSSION}

We found an independent significant association between hip fracture and prolactin-raising antipsychotic medications used prior to the index fracture. The relationship between schizophrenia and hip fracture was confounded by neuroleptics, and the relationship therefore appears to be owing to the effect of neuroleptics rather than diagnosis per se. There is increasing evidence that long-term antipsychotic-induced hyperprolactinaemia is associated with bone mineral density loss which appears to be mediated by secondary hypogonadism (Bilici et al, 2002; O'Keane $\&$ Meaney, 2005). However, ours is the first study, to our knowledge, to demonstrate an association between antipsychotic medication and a clinically relevant outcome of osteoporosis, i.e. fracture 
Table 3 Adjusted odds ratios for hip fractures in men: General Practice Research Database 1987-1999 $(n=9900)$

\begin{tabular}{|c|c|c|c|}
\hline Exposure & OR & $(95 \% \mathrm{Cl})$ & $P$ \\
\hline Schizophrenia diagnosis & 1.61 & $(0.81-3.19)$ & 0.174 \\
\hline Any neuroleptic' & 2.12 & $(1.73-2.59)$ & $<0.001$ \\
\hline \multicolumn{4}{|l|}{ Body mass index ${ }^{2}$} \\
\hline Underweight & 2.51 & $(1.50-4.20)$ & $<0.001$ \\
\hline Overweight & 0.61 & $(0.51-0.74)$ & $<0.001$ \\
\hline Obese & 0.50 & $(0.36-0.69)$ & $<0.001$ \\
\hline Any SSRI & 1.35 & $(1.03-1.77)$ & 0.027 \\
\hline Any laxative & 1.54 & $(1.32-1.78)$ & $<0.001$ \\
\hline Any anticonvulsant & 1.96 & $(1.57-2.45)$ & $<0.001$ \\
\hline Opioid analgesics & 1.54 & $(1.30-1.83)$ & $<0.001$ \\
\hline Cerebrovascular disease & I.7I & $(1.44-2.02)$ & $<0.001$ \\
\hline Blood diseases & 1.66 & (1.39-1.98) & $<0.001$ \\
\hline Non-opioid analgesics & 1.57 & $(I .4 I-I .74)$ & $<0.001$ \\
\hline Any tricyclic & 1.26 & $(1.06-1.49)$ & 0.008 \\
\hline Diseases of intestines & 1.17 & $(1.00-1.36)$ & 0.047 \\
\hline Any hypnotic & 1.26 & $(1.10-1.45)$ & 0.001 \\
\hline Alcohol intake over recommended limit ${ }^{3}$ & 1.50 & $(1.13-2.00)$ & 0.005 \\
\hline \multicolumn{4}{|l|}{ Smoking status ${ }^{4}$} \\
\hline Current smoker & 1.52 & $(1.28-1.80)$ & $<0.001$ \\
\hline Ex-smoker & 0.97 & $(0.77-I .22)$ & 0.772 \\
\hline Hypertensive disease & 0.80 & $(0.69-0.91)$ & 0.001 \\
\hline
\end{tabular}

I. Neuroleptic medication prescribed before or on day of fracture.

2. Compared with normal body mass index, defined as $18.5-24.9 \mathrm{~kg} / \mathrm{m}^{2}$.

3. Alcohol level compared with intake within recommended limits as defined, no more than $2 \mathrm{l}$ units a week for men and 14 units a week for women.

4. Smoking status compared with non-smoker.

of the neck of femur or hip fracture. A history of neuroleptic use was found to be the most significant predictor of an increased risk of hip fracture, with the exception of being underweight.

A number of recent studies have indicated that low bone mineral density is highly prevalent in people with a chronic psychiatric disorder treated with prolactinraising antipsychotic medication (Bilici et al, 2002; Liu-Seifert et al, 2004). Meaney et al (2004) also reported that higher doses of potent typical and atypical antipsychotic medications that block dopamine $\mathrm{D}_{2}$ receptors were associated with increased rates of both hyperprolactinaemia and bone mineral density loss. However, possibly because of a lack of research into the secondary consequences of antipsychotic-induced hyperprolactinaemia, the World Health Organization does not include antipsychotic drugs in its list of prescribed drugs associated with the development of osteoporosis.

Although postmenopausal women are generally most at risk of osteoporosis, possibly owing to low serum oestradiol concentrations (Cummings et al, 1998), we found a higher risk of fracture associated with neuroleptic medication in men. This result is in agreement with several studies of psychiatric patients, which found significantly lower bone mineral density in men than women associated with neuroleptic use (Halbreich et al, 1995; Hummer et al, 2005). These gender differences may be owing to the age differences in onset of schizophrenia (Hafner et al, 1998): men have an age at onset approximately 5 years younger than that in women, and illness-related factors including medication will therefore have had a longer-lasting impact on male patients. An alternative explanation suggested by Hummer \& Huber (2004) is that women with schizophrenia take better care of themselves with regard to adequate nutrition and exercise than men and therefore have less osteoporosis. Unfortunately the data are not available here to test either hypothesis.

There are a number of other known risk factors for osteoporosis among patients with schizophrenia which may be acting as confounders here, including inadequate exercise and exposure to sunshine, poor nutrition, cigarette smoking and polydipsia (Naidoo et al, 2003). Of these, only smoking could be controlled for in this analysis and was found to be a significant factor, but the association was not as strong as with neuroleptic medication. Other mechanisms may also be relevant in causing hip fracture: for example, neuroleptic medications are known to cause sedation, orthostatic hypotension and extrapyramidal side-effects, which may predispose some patients on these treatments to falls (Misra et al, 2004).

We used the GPRD, a large UK primary care data-set, which provided one of the largest data-sets of hip fracture. Like other studies, we found an increased risk of hip fracture to be associated with smoking (Cumming et al, 1997), low body mass index (Farahmand et al, 2000), alcohol intake (for men) (Yuan et al, 2001) and anticonvulsants (Kinjo et al, 2005), and obesity to be protective against hip fracture (Farahmand et al, 2000), giving a high level of face validity to this study.

\section{Limitations of the study}

Although using a large, nationally representative database provides important data from a large sample, detailed clinical information is less available than in smaller studies. Diagnostic categories found on the GPRD are not operationalised and are therefore unlikely to be exactly the same as those found in research or psychiatric practice, and information such as bone mineral density is not available. Lifestyle variables (body mass index, smoking and alcohol intake) are recorded optionally and were missing in a significant number of cases and controls. We created a 'missing' category to enable us to use these fields in the analysis. Residual confounding is therefore possible. Some prescribing of neuroleptics occurs in secondary care only and information on secondary care prescriptions was not available. For this reason details of dosages of antipsychotic medication over time were not reliable, and we categorised neuroleptic exposure as a dichotomous variable (ever/never); some patients might not have received large doses of antipsychotics, and if this were the case the relationship between antipsychotic medication might be owing to mechanisms such as falls, rather than secondary to hyperprolactinaemia. In addition, we could not 
Table 4 Odds ratios for individual antipsychotic medications prescribed before or on day of fracture: General Practice Research Database 1987-1999

\begin{tabular}{|c|c|c|c|c|}
\hline Drug & $\begin{array}{c}\text { Cases, } n \\
(n=16341)\end{array}$ & $\begin{array}{l}\text { Controls, } n \\
(n=29889)\end{array}$ & $\begin{array}{l}\text { Unadjusted } \\
\text { odds ratio }\end{array}$ & $(95 \% \mathrm{Cl})$ \\
\hline \multicolumn{5}{|l|}{ Monotherapy } \\
\hline Chlorpromazine & 270 & 204 & 2.48 & $(2.06-2.99)$ \\
\hline Droperidol & 20 & 5 & 7.65 & $(2.86-20.4 I)$ \\
\hline Flupentixol & 233 & 302 & $\mathrm{I} .43$ & (I.20-I.7I) \\
\hline Fluphenazine & 29 & 16 & 3.34 & $(1.8 I-6.16)$ \\
\hline Haloperidol & 307 & 191 & 2.97 & $(2.47-3.57)$ \\
\hline Pimozide & 19 & 19 & 1.86 & $(0.98-3.53)$ \\
\hline Pipotiazine & 0 & I & & \\
\hline Prochlorperazine & 2100 & 3668 & 1.03 & $(0.97-I .10)$ \\
\hline Promazine & 227 & 134 & 3.26 & $(2.60-4.08)$ \\
\hline Sulpiride & 42 & 26 & 3.00 & $(1.84-4.90)$ \\
\hline Thioridazine & 1403 & 987 & 2.87 & $(2.63-3.13)$ \\
\hline Trifluoperazine & 277 & 248 & 2.06 & $(1.72-2.46)$ \\
\hline Zuclopenthixol & 27 & 14 & 3.36 & $(1.75-6.44)$ \\
\hline \multicolumn{5}{|l|}{ Combination therapy } \\
\hline $\begin{array}{l}\text { Fluphenazine and nor- } \\
\text { triptyline }\end{array}$ & 40 & 98 & 0.74 & $(0.5 \mathrm{I}-\mathrm{I} .07)$ \\
\hline $\begin{array}{l}\text { Trifluoperazine with var- } \\
\text { ious combinations }\end{array}$ & $\mathrm{II}$ & 13 & 1.69 & $(0.76-3.78)$ \\
\hline \multicolumn{5}{|l|}{ Atypical antipsychotics } \\
\hline Amisulpride & 0 & 2 & & \\
\hline Clozapine & 0 & 0 & & \\
\hline Olanzapine & 4 & 3 & 2.67 & $(0.60-11.91)$ \\
\hline Quetiapine & 0 & I & & \\
\hline Risperidone & 21 & II & 3.38 & (I.57-7.24) \\
\hline
\end{tabular}

examine the effect of atypical antipsychotic medications because too few patients had been prescribed them on the GPRD during the exposure period, nor could we examine the effect of individual neuroleptic drugs in a multivariable analysis as there was insufficient statistical power. Larger, more upto-date data-sets could address these issues in the future.

Although case-control studies are prone to bias, one advantage of using a data-set such as the GPRD is that the two major biases in this type of study - selection and recall bias - should be minimised by the prospective collection of data by general practitioners. Reverse causation is normally a possible explanation in casecontrol studies; this possibility should have been excluded here by looking at exposure prior to the occurrence of the first fracture. These findings should also be generalisable.

This study found significant evidence of an association between a diagnosis of schizophrenia and hip fracture, which appeared to be partly explained by neuroleptic medication. This adds to the growing body of evidence of an association between neuroleptic medication and bone mineral density loss. Patients with psychiatric disorders are less likely to have their medical illness diagnosed (Koranyi, 1979; Koran et al, 1989; Redelmeier et al, 1998) and medically managed (Redelmeier et al, 1998), and there is some evidence to suggest that they are less likely to have osteoporosis screened for or treated compared with age-matched control patients (Bishop et al, 2004). If this is the case this has serious public health implications, because the patients who have taken long-term neuroleptic medications are precisely those patients who are probably not being screened for osteoporosis. The evidence base for routinely screening patients prescribed neuroleptics is not available at present and clinicians urgently need more data on who is at highest risk and when (we do not yet know whether psychiatric patients are most at risk of developing osteoporosis after antipsychotic medication is initiated, or after dose-dependent long-term exposure). Randomised controlled trials of interventions to prevent fractures in these patients would enable more effective prophylaxis to be provided by mental health services and by primary care. However, if our findings are replicated, preventive measures should become part of the treatment of patients taking long-term prolactin-raising antipsychotic drugs and may include advice to patients about the importance of a balanced diet containing sufficient amounts of calcium and vitamin $\mathrm{D}$, regular weightbearing exercise, avoidance of tobacco, caffeine and alcohol, and sufficient exposure to sunlight.

\section{ACKNOWLEDGEMENTS}

We thank Professor Richard Hubbard and Christopher Smith, University of Nottingham for their valuable advice and assistance. The study was funded by the Sir Halley Stuart Trust.

\section{REFERENCES}

Abraham, G., Halbreich, U., Friedman, R. H., et al (2003) Bone mineral density and prolactin associations in patients with chronic schizophrenia. Schizophrenia Research, 59, 17-18.

Bilici, M., Cakirbay, H., Guler, M., et al (2002)

Classical and atypical neuroleptics, and bone mineral density, in patients with schizophrenia. International Journal of Neuroscience, II2, 817-828.

Bishop, J. R., Alexander, B., Lund, B. C., et al (2004) Osteoporosis screening and treatment in women with schizophrenia: a controlled study. Pharmacotherapy, 24, 515-521.

Cumming, R. G., Nevitt, M. C. \& Cummings, S. R. (1997) Epidemiology of hip fractures. Epidemiologic Review, 19: 244-257.

Cummings, S. R., Browner, W. S., Bauer, D., et al (1998) Endogenous hormones and the risk of hip and vertebral fractures among older women. New England Journal of Medicine, 339, 733-738.

Farahmand, B. Y., Michaelsson, K., Baron, J., et al (2000) Body size and hip fracture risk. Epidemiology, II, 214-219.

Hafner, H., an der Heiden, H.W., Behrens, S., et al (1998) Causes and consequences of the gender difference in age at onset of schizophrenia. Schizophrenia Bulletin, 24, 99-II3.

Halbreich, U. \& Palter, S. (1996) Accelerated osteoporosis in psychiatric patients: possible pathophysiological processes. Schizophrenia Bulletin, 22, 447-454.

Halbreich, U., Rojansky, N., Palter, S., et al (1995)

Decreased bone mineral density in medicated psychiatric patients. Psychosomatic Medicine, 57, 485-491.

Howard, L. M., Kumar, C., Leese, M., et al (2002) The general fertility rate in women with psychotic disorders. American Journal of Psychiatry, 159, 991-997. 
Hummer, M. \& Huber, J. (2004) Hyperprolactinaemia and antipsychotic therapy in schizophrenia. Current Medical Research Opinion, 20, 189-197.

Hummer, M., Malik, P., Gasser, R.W., et al (2005) Osteoporosis in patients with schizophrenia. American Journal of Psychiatry, 162, 162-167.

Jenkins, R., Lewis, G., Bebbington, P., et al (1997) The National Psychiatric Morbidity surveys of Great Britain - initial findings from the household survey. Psychological Medicine, 27, 775-789.

Kinjo, M., Setoguchi, S., Schneeweiss, S., et al (2005) Bone mineral density in subjects using central nervous system-active medications. American journal of Medicine, II8, 1414

Koran, L. M., Sox, H. C., Marton, K. I., et al (1989) Medical evaluation of psychiatric patients. I. Results in a state mental health system. Archives of General Psychiatry, 46, 733-740.

Koranyi, E. K. (1979) Morbidity and rate of undiagnosed physical illnesses in a psychiatric clinic population. Archives of General Psychiatry, 36, 4I4-419.

Liu-Seifert, H., Kinon, B. J., Ahl, J., et al (2004)

Osteopenia associated with increased prolactin and aging in psychiatric patients treated with prolactinelevating antipsychotics. Annals of the New York Academy of Sciences, 1032, 297-298.

Meaney, A. M., Smith, S., Howes, O. D., et al (2004) Effects of long-term prolactin-raising antipsychotic medication on bone mineral density in patients with schizophrenia. British Journal of Psychiatry, 184, 503-508.

LOUISE HOWARD, PhD, MRCP, MRCPsych, GRAHAM KIRKWOOD, MSc, MORVEN LEESE, PhD, Health Services Research Department, Institute of Psychiatry, Kings College London, UK

Correspondence: Dr Louise Howard, PO 29, Health Services Research Department, Institute of Psychiatry, De Crespigny Park, London SE5 8AF, UK. Email: I. howard@iop.kcl.ac.uk

(First received 28 February 2006, final revision 25 July 2006, accepted I September 2006)

Misra, M., Papakostas, G. I. \& Klibanski, A. (2004) Effects of psychiatric disorders and psychotropic medications on prolactin and bone metabolism. Journal of Clinical Psychiatry, 65, 1607-1618.

\section{Naidoo, U., Goff, D. C. \& Klibanski, A. (2003)}

Hyperprolactinemia and bone mineral density: the potential impact of antipsychotic agents.

Psychoneuroendocrinology, 28 (suppl. 2), 97-108.

Nazareth, I., King, M., Haines, A., et al (1993) Accuracy of diagnosis of psychosis on general practice computer system. BMJ, 307, 32-34.

O'Keane, V. \& Meaney, A. M. (2005) Antipsychotic drugs: a new risk factor for osteoporosis in young women with schizophrenia? Journal of Clinical Psychopharmacology, 25, 26-31.

Redelmeier, D. A., Tan, S. H. \& Booth, G. L. (1998) The treatment of unrelated disorders in patients with chronic medical diseases. New England Journal of Medicine, 338, I516-1520.

van Staa, T. P., Leufkens, H. G. \& Cooper, C. (200I)

Use of inhaled corticosteroids and risk of fractures. Journal of Bone Mineral Research, 16, 58I-588.

Walley, T. \& Mantgani, A. (1997) The UK General Practice Research Database. Lancet, 350, 1097-1099.

World Health Organization (1978) International Statistical Classification of Diseases and Related Health Problems (ICD-9). WHO.

Yuan, Z., Dawson, N., Cooper, G. S., et al (200I) Effects of alcohol-related disease on hip fracture and mortality: retrospective cohort study of hospitalized Medicare beneficiaries. American Journal of Public Health 9l, 1089-1093. 